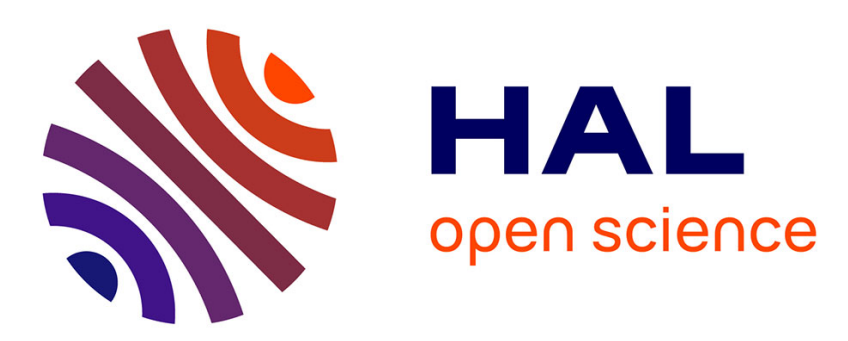

\title{
A new method to study metastable fragmentation of clusters using a reflectron time-of-flight mass spectrometer
}

\author{
J.-M. L'hermite, L. Marcou, F. Rabilloud, P. Labastie
}

\section{- To cite this version:}

J.-M. L'hermite, L. Marcou, F. Rabilloud, P. Labastie. A new method to study metastable fragmentation of clusters using a reflectron time-of-flight mass spectrometer. Review of Scientific Instruments, 2000, 71 (5), pp.2033-2037. 10.1063/1.1150573 . hal-03510627

\section{HAL Id: hal-03510627 \\ https://hal.science/hal-03510627}

Submitted on 13 Jan 2022

HAL is a multi-disciplinary open access archive for the deposit and dissemination of scientific research documents, whether they are published or not. The documents may come from teaching and research institutions in France or abroad, or from public or private research centers.
L'archive ouverte pluridisciplinaire HAL, est destinée au dépôt et à la diffusion de documents scientifiques de niveau recherche, publiés ou non, émanant des établissements d'enseignement et de recherche français ou étrangers, des laboratoires publics ou privés. 


\title{
A new method to study metastable fragmentation of clusters using a reflectron time-of-flight mass spectrometer
}

\author{
J.-M. L'Hermite, ${ }^{\text {a) }}$ L. Marcou, F. Rabilloud, and P. Labastie \\ Laboratoire CAR/IRSAMC (CNRS UMR 5589), Université Paul Sabatier, 118 route de Narbonne, \\ F31062 Toulouse cedex, France
}

(Received 22 November 1999; accepted for publication 1 February 2000)

\begin{abstract}
We propose a new method to study metastable fragmentation of clusters in a reflectron time-of-flight mass spectrometer. This method does not require any modification of the standard apparatus. No preselection of parents is needed: all fragmentation reactions can be analyzed by recording a few numbers of spectra obtained by changing only the repelling voltage of the reflectron $V_{\text {ref }}$. The evolution of a peak as a function of $1 / V_{\text {ref }}$ is a straight line. The extrapolation of this line at $1 / V_{\text {ref }}=0$ gives the mass of the parent $M$ and its slope gives the mass of the fragment $m$ : the whole decay channel set for all parents can be determined unambiguously. The intensity of a peak is stable when scanning $V_{\text {ref }}$ : rather accurate branching ratios can be determined. Application to the fragmentation of clusters containing $\mathrm{Ag}, \mathrm{H}_{2} \mathrm{O}$, and $\mathrm{SF}_{6}$ is presented. Masses are measured to an accuracy of about $0.5 \%$, allowing for example to study independently the dissociation channels involving the two natural isotopes of silver, ${ }^{107} \mathrm{Ag}$ and ${ }^{109} \mathrm{Ag}$. (C) 2000 American Institute of Physics.
\end{abstract}

[S0034-6748(00)04405-1]

\section{INTRODUCTION}

Much can be learned from free cluster fragmentation, and a lot of experiments still use this tool to investigate clusters properties. The aim is to measure decay channels, decay times, and branching ratios, for dissociation occurring either spontaneously or after photo- or collision-induced excitation. From these measurements, it is possible to derive dissociation energies via statistical decay models (for a review, see Ref. 1). Another important application was given by Schmidt et al., who measured the heat capacity of free sodium clusters through the temperature dependence of their photofragmentation mass spectra. ${ }^{2}$ All these experiments concern metastable fragmentation whose time constant ranges from microsecond to a hundred of microseconds. But decay processes with characteristic time of several milliseconds can also be investigated using ion traps. ${ }^{3}$ In all cases, fragments are identified in a mass spectrometer. Several techniques have been used. Early measurements used a double focusing deflection mass spectrometer [massanalyzed ion kinetic energy spectra (MIKES) technique $\left.{ }^{4}\right]$ which provides higher resolution, but is expensive and for which the identification of all decay channels is sequential, i.e., it requires a double scan over both parent and fragment masses.

More widely used are time-of-flight (TOF) techniques. All these techniques can be applied to charged species only, although neutral fragments can be detected in the second method we will describe here.

Both linear TOF(LTOF) and reflectron TOF(RETOF) mass spectrometers have been used. In a TOF clusters are accelerated to a given energy, and their time-of-flight in a free-field region is proportional to the square root of their

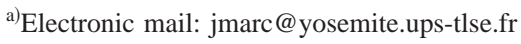

mass. If dissociation occurs in the accelerating region, the fragment arrives at a random time, and is lost. Only fragmentation occurring in the free-field region can be analyzed. Let us first recall the basic LTOF methods. In normal operation mode, undissociated clusters and clusters which undergo dissociation in the free-field region arrive at the same time on the detector (neglecting the recoil energy).

The most simple device consists in eliminating fragments by positioning just before the detector a plate set to a electric potential very close to (just below) the accelerating voltage. ${ }^{5}$ This plate acts as a potential barrier. In this configuration, only the undissociated clusters have enough energy to overcome the barrier, and the comparison of the two spectra with and without the barrier yields a "survival rate," and then the dissociation rate of parent ions. No fragments are identified.

A more efficient selectivity is reached by using two collinear LTOF in tandem: the first one selects the parents, and the second one identifies the fragments. Depletion spectroscopy can be achieved by irradiating the cluster beam with a laser in the second TOF. ${ }^{6}$ A major advantage of this method is that both neutral and ionic fragments can be detected. This method is powerful but is sequential and requires a specific apparatus.

The use of a reflectron offers more flexibility. It has mainly been used in two ways up to now:

The first one is similar to the first LTOF method described above. The reflectron generates a potential barrier and the spectrometer is actually used as a LTOF. In this method, called kinetic energy analysis (KEA), ${ }^{7}$ the time shift of the fragments with respect to their parents is measured. The advantage of this method lies in its simplicity and the simultaneous recording of all decay channels for all clusters. Unfortunately, ambiguities may occur in rich spectra. 


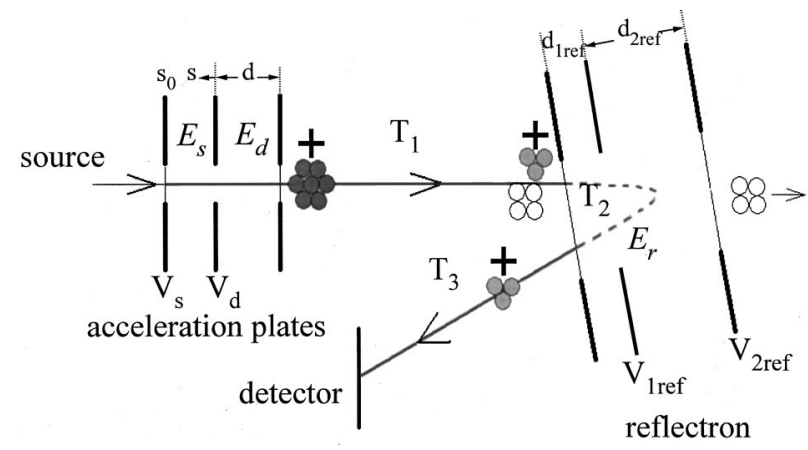

FIG. 1. Schematic view of the fragmentation method.

In the second one, the mass spectrometer is really used as a two-field RETOF mass spectrometer. The time-of-flight of a cluster of a given mass $m$ arriving on the detector depends on whether it comes from the dissociation of a larger cluster of mass $M$ in the free-field drift region or not, and in the case it results from fragmentation, depends on the ratio $m / M$. However, two given reflecting voltage, say $V_{1}$ and $V_{2}$, are able to focus only a small "ratio window" around a nominal ratio $m_{0} / M_{0}$ which varies linearly with $V_{1} / V_{2}$. The observation of all possible fragments then requires to scan the two voltages $V_{1}$ and $V_{2}$, keeping constant the ratio $V_{1} / V_{2}$. Masses are determined from time-of-flight calculation. The ratio $m_{0} / M_{0}$ can also be determined from the value of $V_{1}$ for which the signal vanishes. ${ }^{8}$

We propose a new method which determines (parent, daughter) couples using a single-field reflectron. This method has the advantage of being global (no preselection of parent or scan over parents or fragment is needed), requires a reduced number of recording, and provides an unambiguous determination of decay channel with a good mass resolution.

\section{PRINCIPLE OF THE METHOD}

The experimental setup is shown in Fig. 1. The basic idea is the following: when scanning the voltage of the reflectron $V_{\text {ref }}$, the time-of-flight $T$ of a fragment of mass $m$ evaporated from a parent of mass $M$ can be written $T=T_{1}$ $+T_{2}+T_{3}$ (see Fig. 1). In the zero field region, all clusters move at the speed of the parent, so that $T_{1}$ and $T_{3}$ are proportional to $M^{1 / 2}$. Reflecting time $T_{2}$ is proportional to $v / a$, where $v$ is the entering speed (proportional to $1 / M^{1 / 2}$ ), and $a$ is the acceleration which is proportional to $V_{\text {ref }} / m$. $V_{\text {ref }}$ is set lower than the accelerating voltage in order to avoid reflecting the parents, so that only fragments are detected at the time

$$
T=A M^{1 / 2}+B\left(m / M^{1 / 2}\right) \times 1 / V_{\text {ref }},
$$

where $A$ and $B$ are constant depending on the geometry and the other voltages. By measuring $T$ as a function of $V_{\text {ref }}$ for a given peak, one can deduce both $M$ and $m$. $T$ vs $1 / V_{\text {ref }}$, is a straight line. It is straightforward to deduce $M$ by extrapolating the line at $1 / V_{\text {ref }}=0$, and then $m$ from relation (1).

\section{EXPERIMENTAL SETUP}

The experimental setup (Fig. 1) was reported in previous work. ${ }^{9}$ In normal conditions, clusters are produced in a laser vaporization source in a first chamber, enter the second chamber through a skimmer, and are mass selected in a RETOF mass spectrometer with a two-field reflectron. In this article, we will present results on positive ions produced in the source. In order to accelerate positive ions, the two accelerating voltages $V_{s}$ and $V_{d}$ (see Fig. 1) are pulsed with the help of two fast high voltage switches (Behlke HTS 31 and HTS 51) controlled by two TTL signals generated by a computer via a National Instrument TIO10 device using the LABVIEW package. The two voltages are turned on at the same time. Let us point out that the temporal width of the mass peaks can be much lower than the turn-on rise time of the switches, as easily shown by computer simulation: with a rise time of $70 \mathrm{~ns}$, the width of our peaks at about $1000 \mathrm{amu}$ is less than $20 \mathrm{~ns}$ in normal mode. Ions are detected on two microchannel plates in tandem and the corresponding signal is sent to a $300 \mathrm{MHz}$ digital averaging oscilloscope (Lecroy $9450 \mathrm{~A})$.

For studying metastable fragmentation in the free-field drift region, the two voltages of the reflectron $V_{1 \text { ref }}$ and $V_{2 \text { ref }}$ are set so that $V_{1 \text { ref }} / d_{1 \text { ref }}=\left(V_{2 \text { ref }}-V_{1 \text { ref }}\right) / d_{2 \text { ref }}$, in order to have a homogeneous electric field $E_{r}$ in the whole reflectron which then acts as a single-field reflectron. In the following $V_{\text {ref }}$ refers to $V_{2 \text { ref }}$.

The time-of-flight can be written

$$
\begin{aligned}
T= & T_{1}+T_{2}+T_{3}=\left[2\left(1 / E_{s}-1 / E_{d}\right) X^{1 / 2}+1 / E_{d}\left(X+V_{d}\right)^{1 / 2}\right. \\
& \left.+D\left(X+V_{d}\right)^{-1 / 2}\right](M / q)^{1 / 2}+\left[2 \sqrt{ } 2\left(X+V_{d}\right)^{1 / 2}\right] \\
& \times(m / q) /(M / q)^{1 / 2} \times 1 / E_{r} .
\end{aligned}
$$

$X=s / s_{0} V_{s}$ where $s$ is the initial position of the cluster ion when accelerating voltages $V_{s}$ and $V_{d}$ are turned on and $s_{0}$ its maximal value (see Fig. 1). $s$ is assumed to be constant. It exact value will be discussed later.

$D$ is the total free-field drift length.

The parameters $V_{s}$ and $V_{d}$ are chosen so that time focusing is achieved at the distance $D$. If the length of the reflectron is not too large (i.e., the ratio $E_{d} / E_{r}$ is not too large), the focusing condition is almost that of Wiley and McLaren, ignoring the reflectron, $\partial\left(T_{1}+T_{3}\right) / \partial s=0 .{ }^{10}$ Equation (2) shows that only $T_{2}$, the time spent in the reflectron, depends on $m$. At the limit $E_{r}=\infty$, it is clear that the cluster spends no more time in the reflectron, and then the corresponding time of flight depends only on $M$. On a practical point of view, we proceed as follows:

(1) We record a series of spectra for different values of $V_{\text {ref }}$. $V_{\text {ref }}$ is always kept smaller than $V_{d}$ (see Figs. 2 and 3), in order to avoid pollution with parents contribution whose peaks are very intense and make difficult the identification of small peaks due to fragmented clusters.

(2) We plot the evolution of the time-of-flight for each peak as a function of $1 / V_{\text {ref }}$ (Fig. 2).

(3) We perform a linear regression $f\left(1 / V_{\text {ref }}\right)=\alpha+\beta\left(1 / V_{\text {ref }}\right)$ by least square fit on the corresponding points (Fig. 3).

(4) $M$ is derived from $\alpha$ through the relation (we will assume in the following $q=1$ ) 


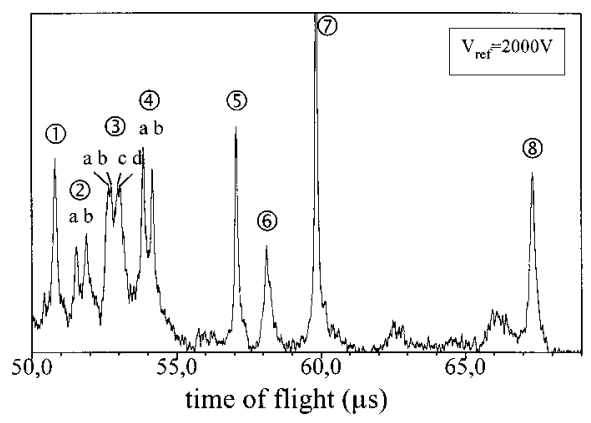

FIG. 2. Fragmentation spectrum for $V_{\text {ref }}=2000 \mathrm{~V}$ (averaged over 500 sweeps).

$$
\begin{aligned}
M= & {\left[\alpha /\left[2\left(1 / E_{s}-1 / E_{d}\right) X^{1 / 2}+1 / E_{d}\left(X+V_{d}\right)^{1 / 2}\right.\right.} \\
& \left.\left.+D\left(X+V_{d}\right)^{-1 / 2}\right]\right]^{2} .
\end{aligned}
$$

$m$ is derived from $\beta$ through the relation

$$
m=\beta\left[2 \sqrt{ } 2\left(X+V_{d}\right)^{1 / 2}\right](M)^{1 / 2} .
$$

With this method, each straight line corresponds to a fragmentation reaction $M \rightarrow m$, not to a parent or a fragment. For this reason, there is no ambiguity on the determination of $M$ and $m$.

The problem of determining exactly the value of $s / s_{0}$ in Eq. (2) is raised especially when studying ions produced in the source. When initially neutral clusters are ionized in the acceleration region with a laser, $s$ is taken as the position of the laser beam. Things become more complicated when ions are formed in the source, so that all ions between the first two plates of the accelerating region are accelerated and may be detected. The time-of-flight at the center of the peak is then determined by the condition $(\partial T / \partial s)_{s 1}=0$ which depends on $E_{r}$. However, in the Wiley-McLaren focusing conditions, it can be shown that the error due to the variation of $s_{f}$ with $E_{r}$ is negligible. Parameters which satisfy the Wiley-McLaren conditions are so that $\left(V_{s}-V_{d}\right) / V_{d} \ll 1$. A first order expansion in $\left(V_{s}-V_{d}\right) / V_{d}$ leads to (assuming $q$ $=1)$ :

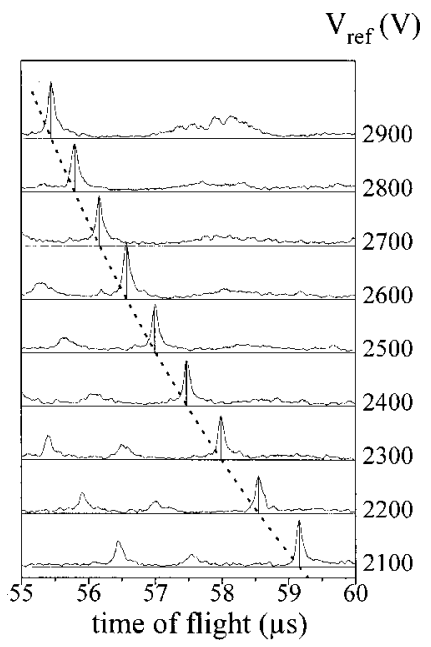

FIG. 3. Peak (7) (see Fig. 4 and Table I) as a function of $V_{\text {ref }}$. The dotted line is the $1 / V_{\text {ref }}$ evolution of the center of the peak [see Eq. (1)].

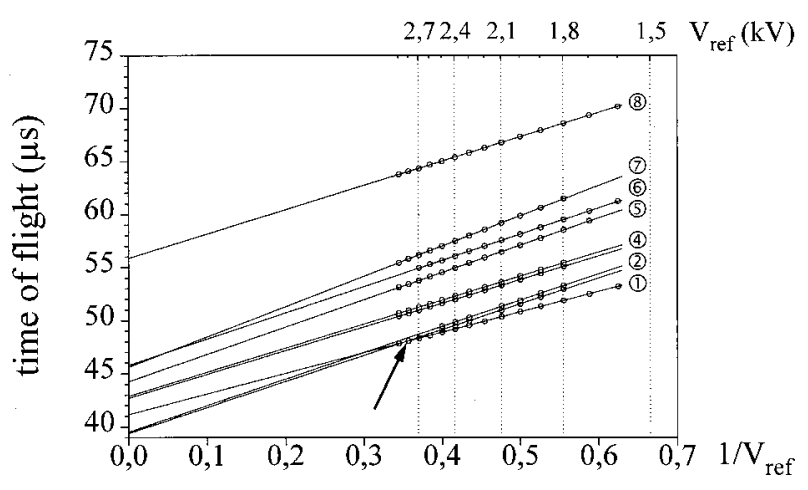

FIG. 4. Least square linear fits of the reactions of Table I. The arrow shows the crossing of the lines corresponding to reactions (1) and (2). At this voltage $(2800 \mathrm{~V})$, it would be impossible to identify independently reactions (1) and (2).

$$
\begin{aligned}
\partial T / \partial s= & (2 M)^{1 / 2}\left[1 / E_{s}-1 / E_{d}+V_{d}^{-1 / 2}\left[(1-D / d) 1 / E_{d}\right.\right. \\
& \left.+2 m / M \quad 1 / E_{r}\right] X-1 / 2 V_{d}^{-3 / 2}\left[(1-3 D / d) 1 / E_{d}\right. \\
& \left.\left.+2 m / M \quad 1 / E_{r}\right] X^{3}\right]=0 .
\end{aligned}
$$

The value of $s$ which verifies Eq. (5) do not depend significantly on $E_{r}$ as far as $d E_{d} /\left(D E_{r}\right) \ll 1$, a condition which is easily satisfied since in the Wiley-McLaren conditions one has $d / D \ll 1$. The error increases as $E_{r}$ decreases (i.e., the length of the reflectron increases). However, even for a $20 \mathrm{~cm}$ length, it remains negligible. For this reason, for a given set of $V_{\text {ref }}$, it is better to apply $V_{\text {ref }}$ to the end plate of the two-field reflectron $\left(V_{2 \text { ref }}=V_{\text {ref }}\right.$ in Fig. 1) rather than to the intermediate plate. In this case, the electric field $E_{r}$ is lower and the time-of-flight shift when scanning $V_{\text {ref }}$ is larger with respect to the width of a peak, thus increasing the resolution of the method.

\section{IMPLEMENTATION AND RESULTS}

We applied the method to clusters containing $\mathrm{Ag}, \mathrm{H}_{2} \mathrm{O}$, and $\mathrm{SF}_{6}$. A silver rod is vaporized with the beam of a Nd:yttrium-aluminum-garnet (YAG) laser (Continuum) doubled at $532 \mathrm{~nm}$ focused by a $25 \mathrm{~cm}$ focal length lens. The carrier gas (helium) contains $\mathrm{SF}_{6}(\approx 10 \%)$ and is slightly contaminated with water. In these conditions, mass spectra reveals the formation of species such as $\left(\mathrm{Ag}_{n} \mathrm{~F}_{n-1}\right)^{+}$, better described as $\left(\mathrm{Ag}^{+} \mathrm{F}^{-}\right)_{n-1} \mathrm{Ag}^{+}$, known to be very stable. ${ }^{11}$ More interesting to us are species containing $\mathrm{H}_{2} \mathrm{O}$ and/or $\mathrm{SF}_{6}$, such as $\left[\mathrm{SF}_{5} \mathrm{H}_{2} \mathrm{O}\left(\mathrm{SF}_{6}\right)_{2}\right]^{+}$, involving van der Waals forces and ion-(induced dipole) interaction, much less stable than the previous ones. ${ }^{12-14}$ These clusters easily undergo spontaneous dissociation in the mass spectrometer and are thus good candidates to test our method. Moreover, the silver atom has two natural isotopes almost equally abundant, ${ }^{107} \mathrm{Ag}$ and ${ }^{109} \mathrm{Ag}$. The ability to separate reactions involving one or the other isotope is a good test for its resolution. Figure 4 shows a representative part of a typical fragmentation spectrum. The identification of the corresponding reactions is given in Table I.

We recorded a series of 19 spectra, varying $V_{\text {ref }}$, by steps $\Delta V_{\text {ref }}=100 \mathrm{~V}$, from 1000 to $2900 \mathrm{~V}$, each of them 
TABLE I. Identification of the fragmentation reactions of Fig. 2.

\begin{tabular}{|c|c|c|}
\hline & Parent & Daughter \\
\hline (1) & $\left(\mathrm{SF}_{3} \mathrm{SF}_{6}\right)^{+}$ & $\mathrm{SF}_{3}^{+}$ \\
\hline \multirow[t]{2}{*}{ (2) } & a $\left({ }^{107} \mathrm{Ag} \mathrm{SF}_{4}\right)^{+}$ & ${ }^{107} \mathrm{Ag}^{+}$ \\
\hline & b $\left({ }^{109} \mathrm{Ag} \mathrm{SF}_{4}\right)^{+}$ & ${ }^{109} \mathrm{Ag}^{+}$ \\
\hline \multirow[t]{5}{*}{ (3) } & a $\left({ }^{107,107} \mathrm{Ag}_{2} \mathrm{X}\right)^{+}$ & ${ }^{107} \mathrm{Ag}^{+}$ \\
\hline & b $\left({ }^{107,109} \mathrm{Ag}_{2} \mathrm{X}\right)^{+}$ & ${ }^{107} \mathrm{Ag}^{+}$ \\
\hline & c $\left({ }^{107,109} \mathrm{Ag}_{2} \mathrm{X}\right)^{+}$ & ${ }^{109} \mathrm{Ag}^{+}$ \\
\hline & d $\left({ }^{109,109} \mathrm{Ag}_{2} \mathrm{X}\right)^{+}$ & ${ }^{109} \mathrm{Ag}^{+}$ \\
\hline & $\mathrm{X}=\mathrm{H}_{2} \mathrm{O}$ or $\mathrm{F}$ & \\
\hline \multirow[t]{2}{*}{ (4) } & a $\left({ }^{107} \mathrm{Ag} \mathrm{SF}_{6}\right)^{+}$ & ${ }^{107} \mathrm{Ag}^{+}$ \\
\hline & b $\left({ }^{109} \mathrm{Ag} \mathrm{SF}_{6}\right)^{+}$ & ${ }^{109} \mathrm{Ag}^{+}$ \\
\hline (5) & $\left(\mathrm{SF}_{5} \mathrm{SF}_{6}\right)^{+}$ & $\mathrm{SF}_{5}^{+}$ \\
\hline (6) & $\left(\mathrm{SF}_{5} \mathrm{H}_{2} \mathrm{O} \mathrm{SF}_{6}\right)^{+}$ & $\mathrm{SF}_{5}^{+}$ \\
\hline (7) & $\left(\mathrm{SF}_{5} \mathrm{H}_{2} \mathrm{O} \mathrm{SF}_{6}\right)^{+}$ & $\left(\mathrm{SF}_{5} \mathrm{H}_{2} \mathrm{O}\right)^{+}$ \\
\hline (8) & {$\left[\mathrm{SF}_{5} \mathrm{H}_{2} \mathrm{O}\left(\mathrm{SF}_{6}\right)_{2}\right]^{+}$} & $\left(\mathrm{SF}_{5} \mathrm{H}_{2} \mathrm{O}\right)^{+}$ \\
\hline
\end{tabular}

averaged over 500 sweeps. The experimental parameters are the following:

(helium $+\mathrm{SF}_{6}$ ) pressure: 2 bar, working frequency: $5 \mathrm{~Hz}$, $s_{0}: 1.0 \mathrm{~cm}, d: 1.5 \mathrm{~cm}, D: 200 \mathrm{~cm}, d_{1 \mathrm{ref}}: 5 \mathrm{~cm}, d_{2 \mathrm{ref}}: 15 \mathrm{~cm}$, $V_{s}: 3200 \mathrm{~V}, V_{d}: 3000 \mathrm{~V}$.

Under these conditions, the complete recording of all spectra took about half an hour (19 spectra $\times 500$ sweeps $\times 0.2 \mathrm{~s}$ ). Let us recall here that all the other methods mentioned in the introducing section implies either a mass-gate preselection of parents (this implies to record one spectrum for each parent) or a scan over the reflectron voltages (it was needed in the last reflectron-based method we described), or both: our method is not more time consuming than the preceding ones. Moreover, let us point out once again that it is the only one which leads to an unambiguous determination of the (parent-daughter ion) couples without preselection of parents.

Figure 2 plotted the evolution of the peak corresponding to reaction (7) when varying $V_{\text {ref }}$. The full width at half maximum (FWHM) of the peaks, of less than $100 \mathrm{~ns}$, does not vary significantly from one voltage to another. The height of the peak is also roughly constant over a voltage scan. These features are interesting since they make possible to compare the relative intensity of the peaks corresponding to different decay channels, leading to reliable relative branching ratios. As mentioned above, the voltages $V_{\text {ref }}$ we used here to identify the reactions do not exceed the lower limit of the accelerating voltage $V_{d}$ in order to avoid contamination of the spectra with ions which did not dissociate. Although we did not do it, it would be possible to follow an identified peak above $V_{d}$ in order to measure the signal of the parent ion which appears for $V_{\text {ref }}>V_{d}$, thus determining the absolute fragmentation rate, i.e., the fraction of the various parent ions which undergo fragmentation.

\section{CALIBRATION}

The set of $(M, m)$ values calculated in a first stage using relations (3) and (4) may differ slightly from expected values (by $\approx 2 \%$ ). Two features are mainly responsible for this. First, the measured time-of-flight $T_{\exp }$ is not exactly the time $T$ of relation (1). Actually, $T_{\exp }=t_{0}+T$, where $t_{0}$ is a small offset due to electronics delay between the time the TTL signal is sent to the high voltage switches and the time high voltages are actually established. It depends also slightly on the trigger level of the oscilloscope. Second, although a good estimated value for $s / s_{0}$ can be chosen with the help of computer simulation, a better value can be found. It is possible to improve the accuracy with a suitable calibration. We proceed as follows: first, the calibration of a high resolution mass spectrum recorded in "normal" mode (a two-field RETOF spectrum) determines $t_{0}$. The value of $t_{0}$ is of course unchanged from normal to "fragmentation" mode. Second, selecting the "best" peaks (i.e., peaks whose assignation is known for obvious chemical reasons) for which $M$ and $m$ are known, the value of $s / s_{0}$ which minimizes the mean error is calculated. Once calibrated in this way, $t_{0}$ and $s / s_{0}$ are fully determined for any experiment with the same values of $V_{s}$ and $V_{d}$. The values for our experimental apparatus are $t_{0}$ $=0.5 \mu \mathrm{s}$ and $s / s_{0}=0.65$. The value of $s / s_{0}$ determines the initial kinetic energy of parents $U_{0}=q\left[V_{d}+\left(V_{s}-V_{d}\right)\right.$ $\times 0.65]=3130 \mathrm{eV}$.

When $V_{\text {ref }}$ is decreased, the peak corresponding to a reaction $M \rightarrow m$ disappears as $V_{\text {ref }}$ becomes lower than $m / M$ $\times U_{0}$ since the fragment is no more reflected in this case. However, even for $V_{\text {ref }}<m / M \times U_{0}$, some fragments with an initial kinetic energy lower than the nominal value $m / M$ $\times U_{0}$ may be reflected, corresponding to parents accelerated from an initial position $s$ between 0 and $0.65 \times s_{0}$. In this case, the shape of the peak is modified, and its center corresponds to a slightly modified value of $s / s_{0}$. The value of $s / s_{0}$ calibrated in the way described above is no more valid for the corresponding points which slightly depart from the straight line defined by Eq. (1). We thus eliminated these points. In our case, since $V_{\text {ref }}$ is stepped by $100 \mathrm{~V}$ units and $V_{s}-V_{d}=200 \mathrm{~V}$, eliminating the two points corresponding to the two lowest values of $V_{\text {ref }}$ for which the peak still appears ensures that all fragments from parents accelerated from $s$ $=0$ to $s=s_{0}$ are able to be reflected. In all the results presented here, these last two points were removed.

\section{RESOLUTION}

Although the resolution of the fragmentation mass spectra is not as good as in normal two-field RETOF mode (in this mode, the resolution of our apparatus is better than 3000 at $1000 \mathrm{amu}$ ), the reactions (2) and (4), involving one silver atom, are clearly split into two components $\mathbf{a}$ and $\mathbf{b}$, corresponding respectively to the ${ }^{107} \mathrm{Ag}$ and ${ }^{109} \mathrm{Ag}$ isotopes (see Fig. 4). The reaction (3) involves two silver atoms, leading to a four-component peak. Fragments containing ${ }^{107} \mathrm{Ag}(\mathbf{a}, \mathbf{b})$ are well distinguished from fragments containing ${ }^{109} \mathrm{Ag}$ $(\mathbf{c}, \mathbf{d})$, but the parents they come from, although well separated in Fig. 4, are sometimes more difficult to determine. For this reaction, we measured the position of the larger peaks constituted respectively of (a and $\mathbf{b})$ and (c and $\mathbf{d})$. This leads only to approximate mean values of $m$ and $M$, the uncertainty on the measure being of course larger in this case than for the other peaks. It is why we were not able to decide whether the parents were $\left(\mathrm{Ag}_{2} \mathrm{H}_{2} \mathrm{O}\right)^{+}$or $\left(\mathrm{Ag}_{2} \mathrm{~F}\right)^{+}$, whose masses differ only by $1 \mathrm{amu}$. However, the binding energy of 


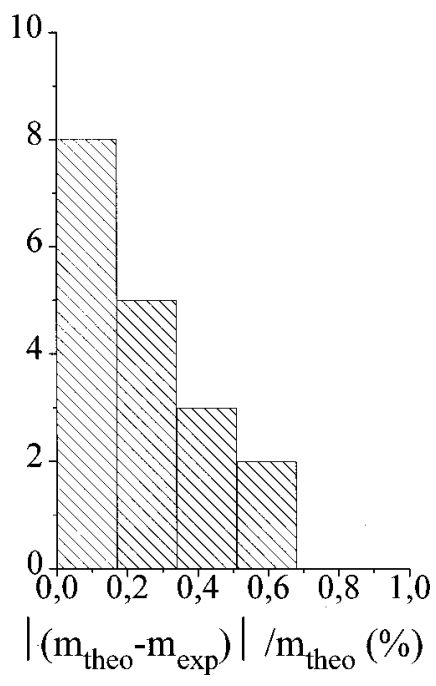

FIG. 5. Histogram of the relative error $\Delta m / m$ including the masses of parents and fragments. 18 masses are taken into account: All reactions of Table I are considered, except reaction (3).

the ionic stoichiometric cluster $\left(\mathrm{Ag}_{2} \mathrm{~F}\right)^{+}$being probably much larger than the one of $\left(\mathrm{Ag}_{2} \mathrm{H}_{2} \mathrm{O}\right)^{+}$, we believe that the reaction with $X=\mathrm{H}_{2} \mathrm{O}$ is more likely to occur. This example shows the limit of resolution of the method.

Figure 5 is a histogram of the relative experimental errors $\left|\left(m_{\text {theo }}-m_{\exp }\right)\right| / m_{\text {theo }}$ where $m_{\text {exp }}$ is the measured value and $m_{\text {theo }}$ the expected value. The histogram includes parents and fragments masses: The uncertainty is less than $0.7 \%$ in our experimental conditions. The resolution depends of course on the number of recorded spectra. The maximum number of points taken into account (limited by the condition $V_{\text {ref }}<m / M \times U_{0}$ and removing the two last points) in Fig. 3 is 14 . This number decreases as $m / M$ decreases. For a given $\Delta V_{\text {ref }}$ from one spectrum to another, the number of points and then the resolution decreases: the method is more accurate for "small" fragments.

${ }^{1}$ Walt A. de Heer, Rev. Mod. Phys. 65, 611 (1993).

${ }^{2}$ M. Schmidt, R. Kusche, W. Kronmüller, B. von Issendorf, and H. Haberland, Phys. Rev. Lett. 79, 99 (1997).

${ }^{3}$ U. Hild, G. Dietrich, S. Krückeberg, M. Lindinger, K. Lützenkirchen, L. Schweikhard, C. Walther, and J. Ziegler, Phys. Rev. A 57, 2786 (1998).

${ }^{4}$ P. G. Lethbridge and J. Stace, J. Chem. Phys. 89, 4062 (1988).

${ }^{5}$ O. Echt, D. Kreisle, M. Knapp, and E. Recknagel, Chem. Phys. Lett. 108, 401 (1984).

${ }^{6}$ C. Brechignac, P. Cahuzac, F. Carlier, and J. Leygnier, Chem. Phys. Lett. 164, 433 (1989).

${ }^{7}$ C. Guillaume, J. Le Calvé, I. Dimicoli, and M. Mons, Z. Phys. D: At., Mol. Clusters 32, 103 (1994).

${ }^{8}$ H. Kühlewind, H. J. Neusser, and E. W. Schlag, Int. J. Mass Spectrom. Ion Phys. 51, 255 (1983).

${ }^{9}$ P. Labastie, J.-M. L'Hermite, Ph. Poncharal, and M. Sence, J. Chem. Phys. 103, 6362 (1995).

${ }^{10}$ W. C. Wiley and I. H. McLaren, Rev. Sci. Instrum. 26, 1150 (1955).

${ }^{11}$ I. Rabin, C. Jackschath, W. Schulze, and F. W. Froben, Z. Phys. D: At., Mol. Clusters 19, 401 (1991).

${ }^{12}$ D. Feller, E. D. Glendening, and W. A. de Jong, J. Chem. Phys. 110, 1475 (1999).

${ }^{13}$ T. A. Beu, Y. Okada, and K. Takeuchi, Eur. J. Phys. 6, 99 (1999).

${ }^{14}$ J. F. Winkel, A. B. Jones, C. A. Woodward, D. A. Kirkwood, and A. J. Stace, J. Chem. Phys. 101, 9436 (1994). 